\title{
Science China Materials enters its sixth year
}

\author{
Guilin Wang ${ }^{1}$ and Yadong $\mathrm{Li}^{2^{*}}$
}

As Science China Materials (SCMs) enters the sixth year, we take this occasion to look back its development in the past five years, especially in 2019. Much has been achieved, and yet we should start by thanking our authorship and readership, both of which are the base for the growth of SCMs.

It has been an exciting and worthy journey making SCMs one of the prestigious journals in materials science on an international basis. An important progress in its rapid growth is that SCMs has ranked as Q1 (top 25\%) journal in the Journal Citation Reports (JCR) for three consecutive years, with its impact factor jumping from 3.956 in 2016 to 5.636 in 2018 (Fig. 1). The total number of citations of SCMs has increased exponentially, reaching 1878 in 2018, and it is expected to approach 3000 in 2019.

The numbers of submissions and published articles have also continuously increased. We published 187 articles with the submissions around 700 in 2019. The published papers took $\sim 1936$ pages, with a $19 \%$ increase from 1628 pages in 2018 (Fig. 2). The growth of the journal in terms of page numbers can be considered a token of its success. Despite the doubling in volume in five years, we have not compromised our high standards. SCMs is still a relatively small and highly selective journal. The rejection rate for manuscripts submitted in 20182019 was $70 \%-80 \%$, up from $58 \%$ in 2017 . In addition, the number of unsolicited papers published in 2019 has a more drastic increase by $48 \%$ (from 103 to 152), compared with the increase in the total number of published articles (8\%, from 174 to 187 ).

As a general materials science journal, SCMs has continued to show significant advances in every area of this subject, and the contributions come from authors in a broad range of disciplines, all with the same goal of achieving efficient, practical and environment-friendly materials. We believe one of the many important roles that SCMs can play is to help combine the efforts of different disciplines, including chemistry, physics, engineer- ing, and biomedical sciences. Herein, we list a couple of highly cited and most significant review papers published in SCMs since its launch: (i) "Design and fabrication of semiconductor photocatalyst for photocatalytic reduction of $\mathrm{CO}_{2}$ to solar fuel" authored by Li et al. [1] in 2014, selected as ESI highly cited paper with 240 citations; (ii) "Beyond Li-ion: electrode materials for sodium- and magnesium-ion batteries" authored by Massé et al. [2] in 2015, cited by 135 times; (iii) "Science and technology in high-entropy alloys" authored by Zhang et al. [3] in 2018, selected as ESI highly cited paper with 107 citations. Some interesting research articles published in 2018-2019 are also worthy of being noted (see Refs [4-10]).

For international distribution of SCMs, we continue the partnership with Springer Nature. The number of fulltext downloads has been rising markedly, and is expected to reach $\sim 150,000$ in 2019 , up from 110,000 in 2018 . The WeChat public account of SCMs has been running more
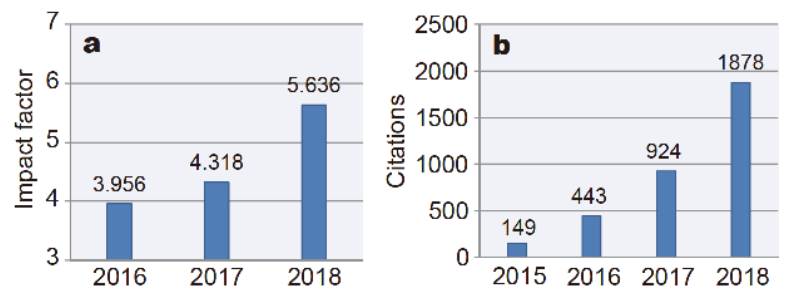

Figure 1 Increase in impact factor (a) and total ciations (b) of SCMs in 2015-2018.
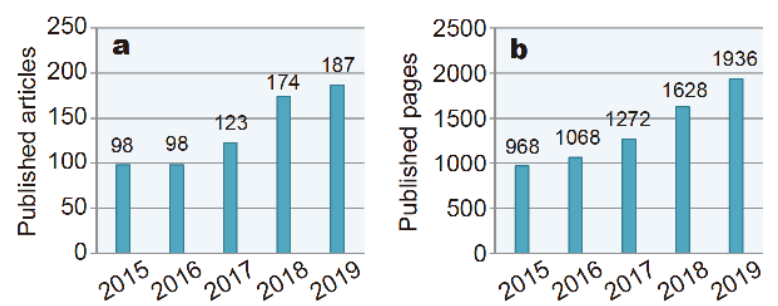

Figure 2 Increase in size of SCMs: published articles (a) and pages (b) in 2015-2019.

\footnotetext{
${ }^{1}$ Managing Editor of SCMs. Science China Press, Beijing 100171, China

${ }^{2}$ Editor-in-Chief of SCMs. Department of Chemistry, Tsinghua University, Beijing 100084, China

${ }^{*}$ Corresponding author (email: ydli@tsinghua.edu.cn)
} 


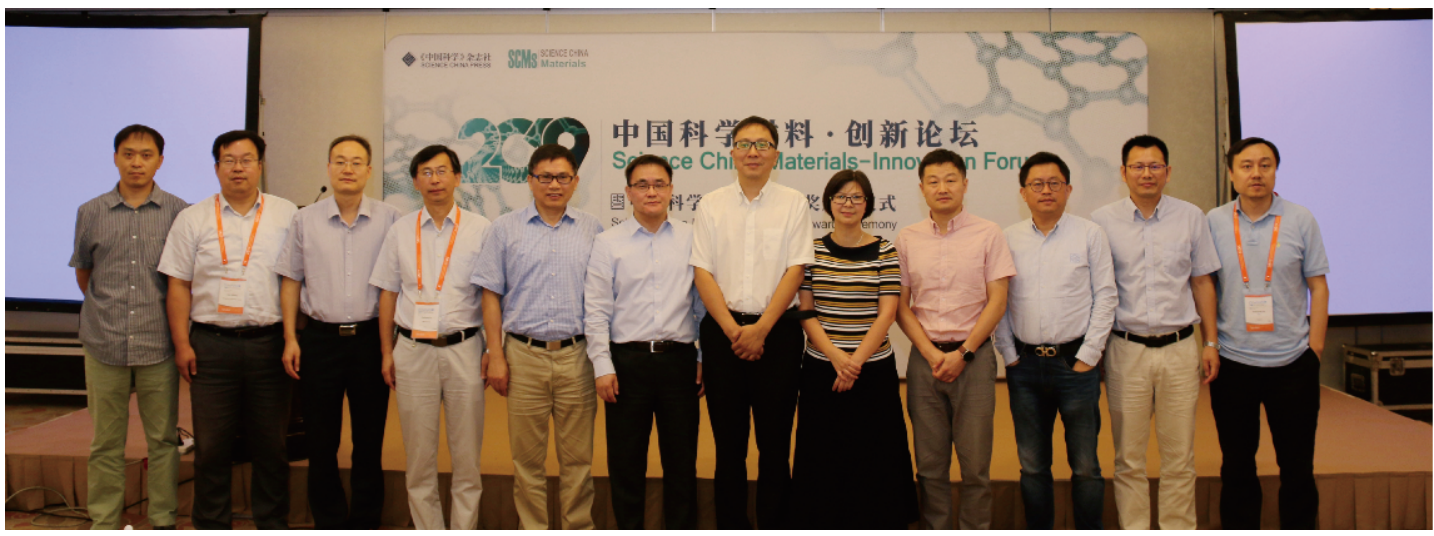

Figure 3 Photograph of the lectures and chair (Xun Wang) of the 1st Science China Materials-Innovation Forum with the Editor-in-Chief, Yadong Li (from left to right: Jianhui Hou, Xun Wang, Liangti Qu, Shu-Hong Yu, Yadong Li, Hong Yang, Xiangfeng Duan, Yu Huang, Dayong Jin, Quan-Hong Yang, Huisheng Peng and Xiaogang Liu).

actively, playing an important role in timely disseminating the latest published papers in SCMs and effectively promoting their visibility. It has gotten more than 11,000 followers so far.

In August 2019, SCMs successfully held an Innovation Forum. The Forum was organized as a one-day session with 14 invited lectures presented by the distinguished Editorial Board members of SCMs together with other leading scientists in the field of materials science (Fig. 3). In addition, the winner of the 2019 Science China Materials-Innovation Award was announced to be Professor Xiangfeng Duan from the University of California, Los Angeles. The Innovation Forum will be held at least once a year, with the aim to showcase exceptionally high quality and cutting-edge research results and perspectives on future directions of some forefront areas of materials science, and to build up the strong relationship between SCMs and the scientific community.

The continued growth and success of SCMs are largely attributed to the contributions and support of the Editorial and Editorial Advisory Boards. Many of them are deeply involved in soliciting high-quality papers, screening and reviewing manuscripts, and promoting the journal all over the world. The efficient handling of submitted manuscripts is mainly due to the efforts of the Associate Editors, including Profs Xun Wang, Guozhong Cao, Jun Chen, Hui-Ming Cheng, Shu-Hong Yu and Xueyuan Chen. Three editors in the Editorial Office, Drs Guilin Wang, Shaohua Dong and Cancan Li, have endeavored to handle the copy-editing and proof correction, and are largely responsible for the healthy production pipeline and high technical quality of the journal.

As the Editorial team, we thank all the authors, referees and readers for their continuing support to SCMs. We promise the continued growth of the journal, driving its impact factor higher while ensuring a strict and fair peer review process and a short submission-to-publication timeline for all valuable research resulting from those devoted to materials science across the world.

Received 19 November 2019; accepted 20 November 2019; published online 25 November 2019

1 Li X, Wen J, Low J, et al. Design and fabrication of semiconductor photocatalyst for photocatalytic reduction of $\mathrm{CO}_{2}$ to solar fuel. Sci China Mater, 2014, 57: 70-100

2 Massé RC, Uchaker E, Cao G. Beyond Li-ion: electrode materials for sodium- and magnesium-ion batteries. Sci China Mater, 2015, 58: 715-766

3 Zhang W, Liaw PK, Zhang Y. Science and technology in highentropy alloys. Sci China Mater, 2018, 61: 2-22

4 Li J, Ma H, Xie Q, et al. Topological quantum catalyst: Dirac nodal line states and a potential electrocatalyst of hydrogen evolution in the TiSi family. Sci China Mater, 2018, 61: 23-29

5 Liu G, Jin W. Graphene oxide membrane for molecular separation: challenges and opportunities. Sci China Mater, 2018, 61: 1021-1026

6 Zhu Y, Cao T, Li Z, et al. Two-dimensional $\mathrm{SnO}_{2} /$ graphene heterostructures for highly reversible electrochemical lithium storage. Sci China Mater, 2018, 61: 1527-1535

7 Liu J, Guo X, Lin Q, et al. Excellent ductility and serration feature of metastable $\mathrm{CoCrFeNi}$ high-entropy alloy at extremely low temperatures. Sci China Mater, 2019, 62: 853-863

8 Guo R, Yao S, Sun X, et al. Semi-liquid metal and adhesion-selection enabled rolling and transfer (SMART) printing: A general method towards fast fabrication of flexible electronics. Sci China Mater, 2019, 62: 982-994

9 Zheng S, Zeng M, Cao H, et al. Insight into the rapid growth of graphene single crystals on liquid metal via chemical vapor deposition. Sci China Mater, 2019, 62: 1087-1095

10 Shao YT, Wang ZC, Li BZ, et al. $\mathrm{BaTh}_{2} \mathrm{Fe}_{4} \mathrm{As}_{4}\left(\mathrm{~N}_{0.7} \mathrm{O}_{0.3}\right)_{2}$ : An ironbased superconductor stabilized by inter-block-layer charge transfer. Sci China Mater, 2019, 62: 1357-1362 